Journal of Applied Pharmaceutical Science Vol. 2 (10), pp. 026-030, October, 2012

Available online at http://www.japsonline.com

DOI: 10.7324/JAPS.2012.21005

ISSN 2231-3354 (cc) BY-NC-SA

\title{
Anti-ischemic effect of ethyl acetate extract of Aquilaria crassna by attenuation of p38-MAPK activation
}

\author{
Chanyatip Suwannasing ${ }^{1.2}$, Nitchawat Paiyabhroma ${ }^{1,3}$, Sarawut Kumphune (3* $^{1, *}$ \\ ${ }^{1}$ Biomedical Research Unit in Cardiovascular Sciences (BRUCS), Faculty of Allied Health Sciences, Naresuan University, Phitsanulok 65000, Thailand. \\ ${ }^{2}$ Department of Radiological Technology, Faculty of Allied Health Sciences, Naresuan University, Phitsanulok 65000, Thailand. \\ ${ }^{3}$ Department of Medical Technology, Faculty of Allied Health Sciences, Naresuan University, Phitsanulok 65000, Thailand.
}

\section{ARTICLE INFO}

Article history:

Received on: $15 / 08 / 2012$

Revised on: 29/08/2012

Accepted on: 05/09/2012

Available online: 28/10/2012

Key words:

Ischemic Heart Disease;

Aquilaria crassna; myocardial

ischemia/reperfusion; infarct

volume; $p 38$ MAPK

\section{INTRODUCTION}

Myocardial ischemia exists when the reduction of the coronary flow is so severe that the supply of oxygen to the myocardium is inadequate for the oxygen demands of the tissue, resulting in the accumulation of metabolites in the ischemic region. Severe and prolonged ischemia ultimately results in cellular necrosis (Jennings \& Reimer, 1991). Currently, the most efficient way to reduce aggravation of the disease is to achieve rapid reperfusion (Braunwald, 1996). Numerous intracellular signaling pathways play an important role in the myocardial response to ischemia/reperfusion injury.

\footnotetext{
* Corresponding Author

Sarawut Kumphune

Biomedical Research Unit in Cardiovascular Sciences (BRUCS) and Department of Medical Technology, Faculty of Allied Health Sciences, Naresuan University, Phitsanulok, 65000, Thailand.

Tel.: +66(0) 83-960 6006; Fax: +66(0) 55966300
}

In particular, p38 MAPK has been widely investigated in this issue. Pre-clinical investigation indicated that inhibition of p38 MAPK activation could reduce myocardial injury, suggesting the therapeutic potential of p38 MAPK inhibitors in ischemic heart disease (Kumphune et al., 2012 ${ }^{\mathrm{a}}$ ). Aquilaria crassna Pierre ex Lecomte or agarwood is heartwood of tropical tree belongs to the family Thymelaeaceae and class Magnoliosida (Dash et al., 2008), which can be found in many countries throughout the oriental region. It has been used as folk medical treatment for treatment of cardiac disorders (Miniyar et al., 2008). Interestingly, in Thailand, A. crassna extract has been using as the ingredient of Ya-hom, a traditional Thai herbal formulation for the treatment of fainting (Suvitayavat et al., 2005), by targeting the cardiovascular system. Our previous finding demonstrated the anti-inflammatory 
effect of ethyl acetate extract of A. crassna on tumor necrosis factor alpha (TNF- $\alpha$ ) expression by attenuating p38 MAPK activation (Kumphune et al., 2011).

Recently, we reported that $5 \mathrm{mg} / \mathrm{ml}$ of $A$. crassna extract could reduce simulated ischemia induced cell death in cardiac myoblast cell line, H9c2 (Jermsri et al., 2012 ${ }^{\mathrm{a}}$ ), as well as isolated adult rat ventricular myocytes (ARVMs) (Kumphune et al., $2012^{\mathrm{b}}$ ). The findings from these previous studies showed that the A. crassna extract could inhibit ischemia-induced p38 MAPK activation.

Moreover, Jermsri et al. reported the cytoprotective effect of A. crassna extract on actin cytoskeleton organization, in cardiac cell subjected to simulated ischemia (Jermsri et al., 2012 ${ }^{\mathrm{b}}$ ). However, the effect of this plant extract on infarct size still needs to be investigated. Therefore, the present work is designed to study the effect of A. crassna crude extract on infarct volume and its mechanism on p38 MAPK activation.

\section{MATERIALS AND METHODS}

\section{Plant Material and extraction}

Aquilaria crassna Pierre ex Lecomte was obtained from

Mr. Choosak Rearngrattanabhume. The plant was originally cultivated at the area in Pong Nam Ron district, Chantaburi province, Thailand. The specimens were collected on September 2010 and subsequently identified by Dr. Pranee Nangngam, department of biology, faculty of science, Naresuan University.

The specimen voucher number 002540 was kept at department of biology herbarium, faculty of science, Naresuan University. The heartwood was sliced into small pieces, left air died, and extracted by the methods described in the previous study (Kumphune et al., 2011).

\section{Animals}

Adult ICR mice (6-8 weeks or body weight greater than $25 \mathrm{~g}$ ) were purchased from National Laboratory Animal Center, Mahidol University, Bangkok, Thailand. The animals were housed in a room maintained at $23 \pm 2{ }^{\circ} \mathrm{C}$ and a relative humidity of $50 \%$ with $12 \mathrm{~h}: 12 \mathrm{~h}$ of dark: light cycle, at center for animal research, Naresuan University, Phitsanulok, Thailand for at least 2 weeks before performing the experiments. The study protocol was approved by Naresuan University Animal Ethics committee (protocol license No.55 04 0005).

\section{Retrograde perfusion of isolated murine heart}

All male mice were anesthetized by intraperitoneal injection (IP) with pentobarbital $(300 \mathrm{mg} / \mathrm{kg}$ ) and heparin $(150$ units). The hearts were rapidly isolated and placed in ice cold modified Krebs-Henseleit (K-H) buffer (18.5 mM of $\mathrm{NaCl}, 25.0$ $\mathrm{mM}$ of $\mathrm{NaHCO}_{3}, 4.75 \mathrm{mM}$ of $\mathrm{KCl}, 1.18 \mathrm{mM}$ of $\mathrm{KH}_{2} \mathrm{PO}_{4}, 1.19 \mathrm{mM}$ of $\mathrm{MgSO}_{4}, 11.0 \mathrm{mM}$ of D-glucose, and $1.4 \mathrm{mM}$ of $\mathrm{CaCl}_{2}$ ). The aorta was cannulated on a Langendorff apparatus and retrograde-perfused at a constant pressure with $\mathrm{K}-\mathrm{H}$ buffer equilibrated with $95 \% \mathrm{O}_{2}$ and $5 \% \mathrm{CO}_{2}$ at $37{ }^{\circ} \mathrm{C}$. The hearts were randomized to $5-\mathrm{mg} / \mathrm{ml}$ ethyl acetate extract of Aquilaria crassna (A.E) for $30 \mathrm{~min}$ prior to ischemia with blinding to the corresponding vehicle ( $0.001 \%$ Dimethysulfoxide). Infarction was caused by $30 \mathrm{~min}$ of global ischemia followed by $2 \mathrm{~h}$ of reperfusion and delineated by $1 \%$ triphenyltetrazolium chloride (TTC) (Sigma, St. Louis, MO, USA) (Fig.1A).

\section{Infarct volume assessment in isolated murine heart}

The infarction assessment in Isolated Murine Hearts was performed as previously described (Kumphune et al., 2010). After 2 hours of reperfusion, hearts were perfused for 1 min with $5 \mathrm{ml}$ of $1 \%(\mathrm{w} / \mathrm{v}) \mathrm{TTC}$, then removed and placed in $1 \%$ TTC solution at $37^{\circ} \mathrm{C}$ for $10 \mathrm{~min}$. The hearts were incubated in $2.5 \%$ glutaraldehyde for 1 minute, and set in 5\% agarose before sectioning in $750 \mu \mathrm{m}$ thick slices. All slices were incubated in $10 \%$ $(\mathrm{v} / \mathrm{v})$ formaldehyde overnight at room temperature before rehydration overnight phosphate buffer saline (PBS) at $4^{\circ} \mathrm{C}$.

The heart sections were scanned and planimetry was carried out and surface area of the whole, and TTC-negative, myocardium was transformed to volume. The TTC-negative infarction volume was expressed as a percentage of heart volume. All analyses of infarct size were done by an investigator, who was blinded with regard to the group assignments.

\section{Measurement of p38 MAPK MAPK activation by western blot analysis}

The hearts were subjected to $30 \mathrm{~min}$ of stabilization by perfusion with modified K-H buffer and then randomly exposed to $5-\mathrm{mg} / \mathrm{ml}$ the extract or vehicle control $(0.001 \%$ DMSO) for $30 \mathrm{~min}$ prior to $10 \mathrm{~min}$ of global ischemia.

Then the hearts were rapidly snap frozen (Fig.1B). The heart tissues were homogenized and solubilized in SDSPolyacrylamide gel electrophoresis (SDS-PAGE) sample buffer as described previously (Kumphune, 2010).

The heart proteins was separated on $10 \%$ SDSpolyacrylamide gels; transferred to polyvinylidene difluoride (PVDF) membranes, and probed overnight at $4{ }^{\circ} \mathrm{C}$ with 1:1000 total p38 MAPK or diphospho-p38 MAPK antibody (Santa Cruz Biotechnology Inc., CA, USA). Bands corresponding to the detected protein of interest were developed by autoradiographic method. Band densities were quantified and express as fold phosphorylation (Phospho:Total p38 MAPK).

\section{Statistical analysis}

All values are expressed as Mean \pm S.D. All comparisons involving more than one group were assessed for significance using one-way analysis of variance (ANOVA) with Tukey post hoc test. A statistical value of less than 0.05 was considered significant. 


\section{A}

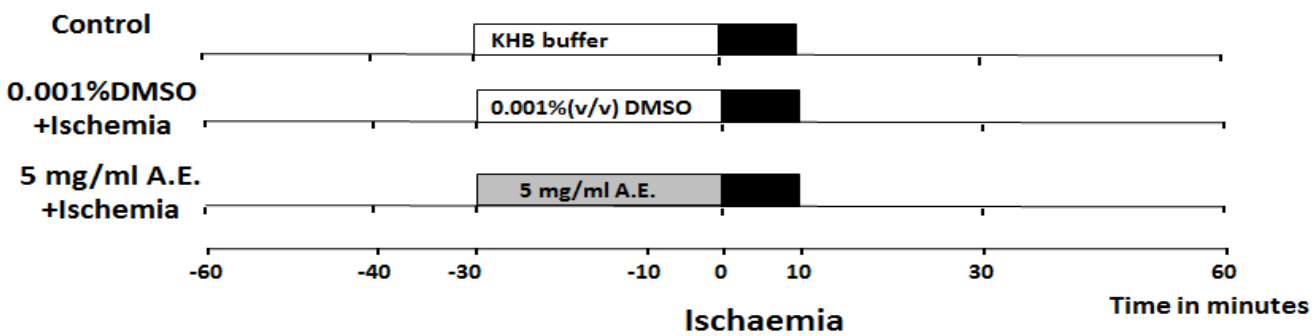

B

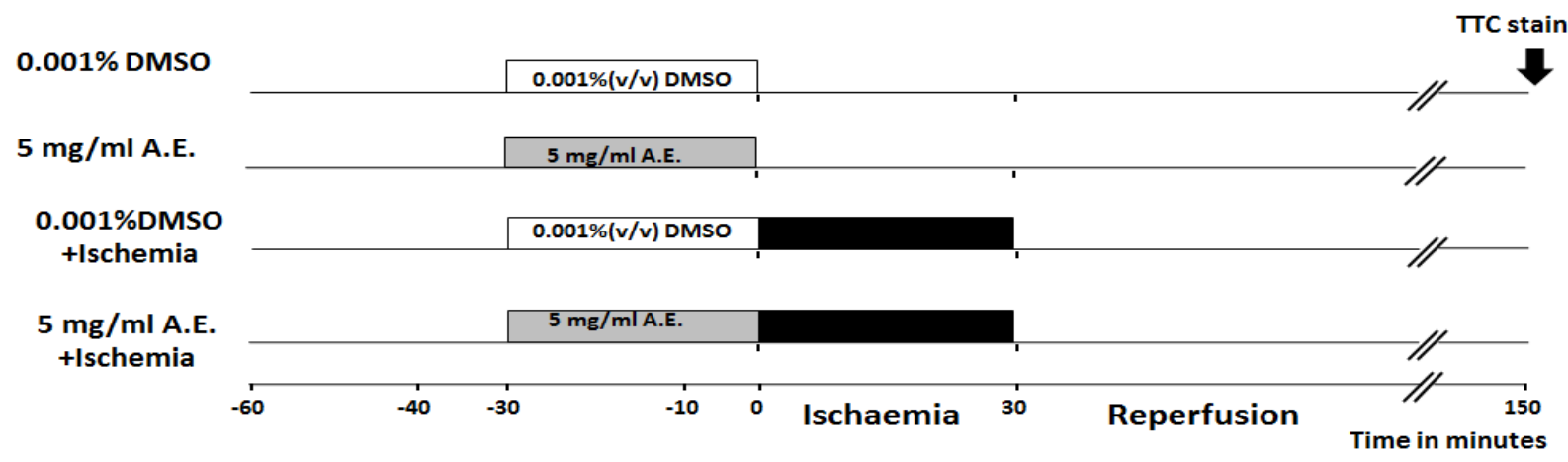

Fig. 1: Schematical representation of isolated mouse heart perfusion protocol used to assess the effect of ethyl acetate extract of A. crassna (A.E.) on p38 MAPK MAPK phosphorylation and the sensitivity to infarction. (A) Isolated mouse hearts were subjected 40 min stabilization with K-H buffer before exposing to 10 min ischemia, in the presence and absence of pre-treatment with $5-\mathrm{mg} / \mathrm{ml} \mathrm{A.E.,} \mathrm{or} \mathrm{vehicle} \mathrm{control} \mathrm{(0.01 \%} \mathrm{DMSO),} \mathrm{for} 30$ min. (B) Infarct size determination, The heart samples were isolated mouse hearts were stabilized with K-H buffer for $30 \mathrm{~min}$, before subjected to 30 min global ischemia, in the presence and absence of $5-\mathrm{mg} / \mathrm{ml} \mathrm{A.E.,} \mathrm{or} \mathrm{vehicle} \mathrm{control} \mathrm{(0.001 \%} \mathrm{DMSO).} \mathrm{At} \mathrm{the} \mathrm{end} \mathrm{of} \mathrm{reperfusion,} \mathrm{the} \mathrm{hearts} \mathrm{were} \mathrm{perfused} \mathrm{with} 1 \%$ TTC for $1 \mathrm{~min}$, and the samples were collected from infarct size analysis.

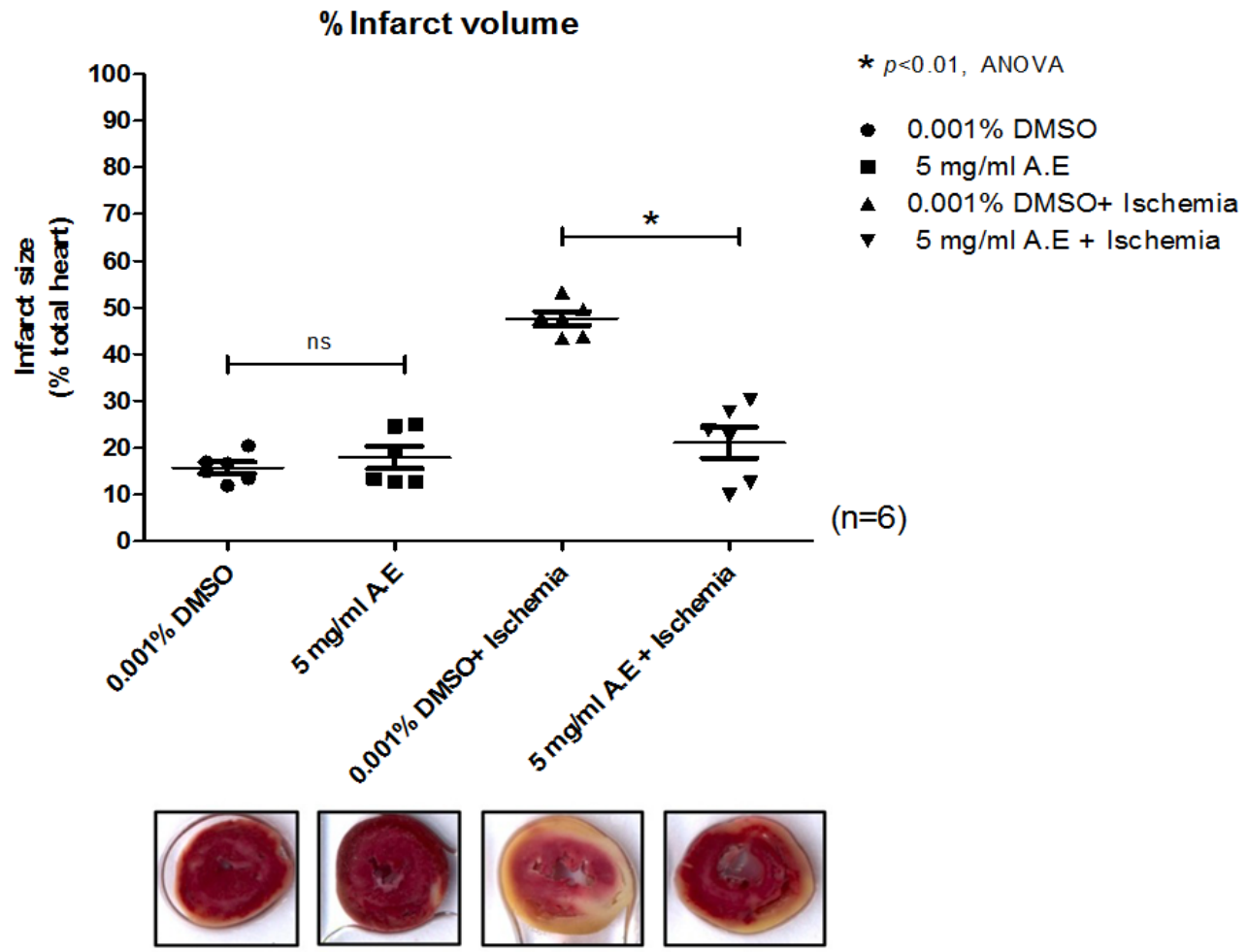

Fig. 2: The sensitivity to myocardial infarction in murine hearts subjected to global ischemia/reperfusion, in the presence and absence of $A$. crassna extract.The data shown is the normalized infarction volume of isolated murine hearts in the presence of $5-\mathrm{mg} / \mathrm{ml} \mathrm{A.E.,} \mathrm{or} \mathrm{vehicle}(0.001 \%$ DMSO). ns, not significant; * $p<0.01$ vs group, (one way ANOVA, $\mathrm{n}=6$ ). 

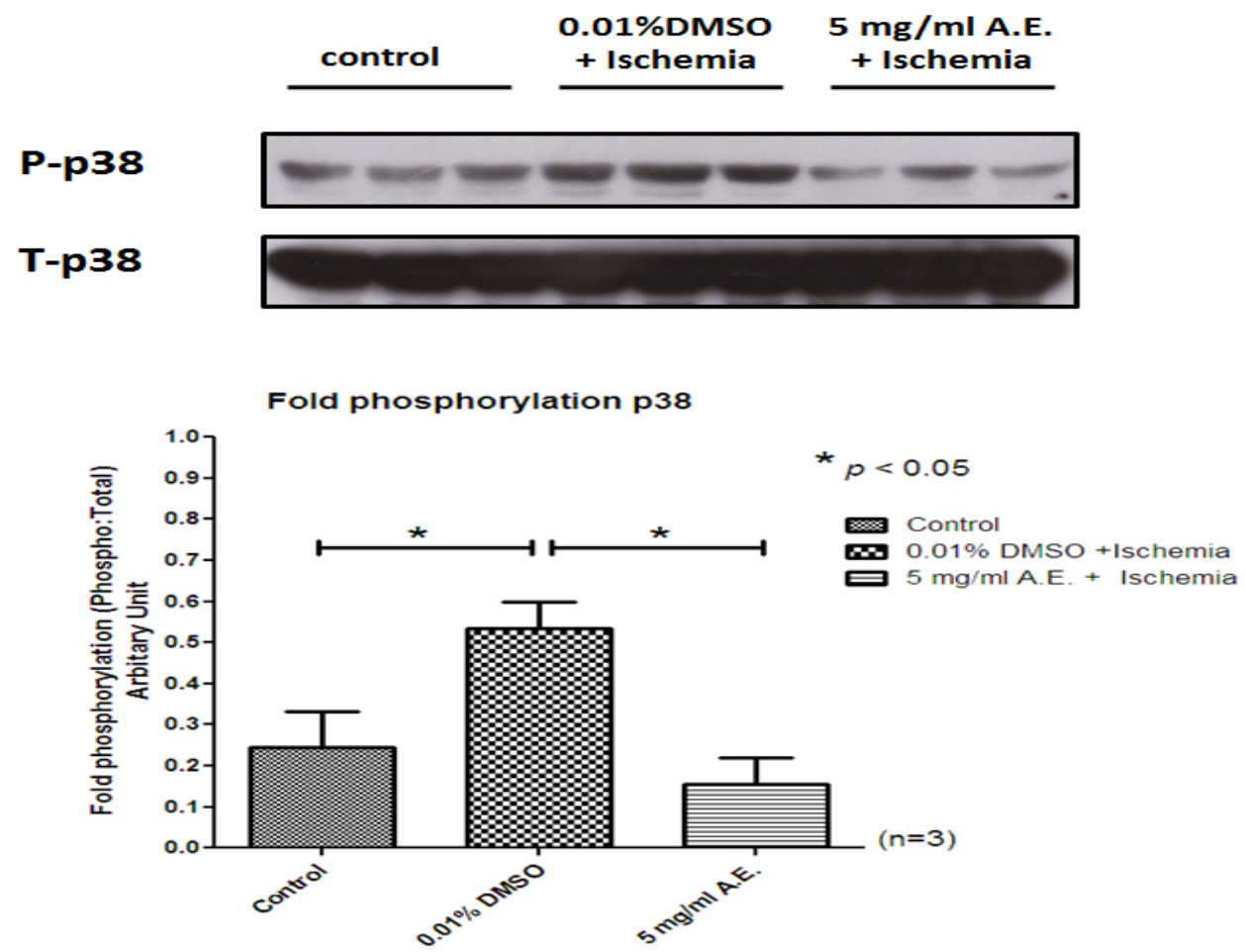

Fig. 3: p38 MAPK MAPK activation during myocardial Ischemiain hearts exposed to pre-treatment of $A$. crassna extract. Isolated hearts were subjected to $10 \mathrm{~min}$ of ischemia in the presence and absence of 5-mg/ml A.E., or vehicle (0.001\% DMSO). The quantification of band density was expressed as Mean \pm S.D. $* p<0.05$ vs group, (one way ANOVA, $\mathrm{n}=3$ ).

\section{RESULTS}

The effect of ethyl acetate extract of Aquilaria crassna on sensitivity to myocardial infarction in isolated murine heart

We examined the sensitivity to infarction of isolated murine hearts in response to $30 \mathrm{~min}$ of global ischemia, in the presence and absence of $5-\mathrm{mg} / \mathrm{ml}$ ethyl acetate extract of Aquilaria crassna (A.E.; Aquilaria Extract). The concentration of 5-mg/ml used in the whole experiments is the concentration that previously showed cardioprotective in in vitro model of H9c2 (Jermsri et al., $2012^{\mathrm{a}}$ ) and isolated adult rat ventricular myocytes (Kumphune et al., 2012 $2^{\mathrm{b}}$ ).The results showed that isolated murine hearts perfused with $5-\mathrm{mg} / \mathrm{ml}$ of A.E. or vehicle control (0.001\% DMSO) for 30 min on Langendorff perfusion system caused small and nonsignificant infarct volume $(15.73 \pm 2.99 \%$ versus $17.93 \pm 5.866$ $\%$, respectively). However, 30 min of global ischemia caused greater in infarct volume. Pre-treatment of the heart with $5-\mathrm{mg} / \mathrm{ml}$ of A.E. for $30 \mathrm{~min}$ prior to global ischemia significantly reduced infarct volume $(47.61 \pm 3.68 \%$ versus $21.08 \pm 8.16 \%, p<0.01)$ (Fig. 2)

The effect of ethyl acetate extract of Aquilaria crassna on p38 MAPK Dual Phosphorylation during Myocardial Ischemia

To determine whether the reduction of infarct size in heart pre-treated with the extract involving the inhibition of p38 MAPK as the dominant mechanism, we examined p38 MAPK phosphorylation in the presence and absence of $5-\mathrm{mg} / \mathrm{ml}$ A.E. The results showed that $\mathrm{p} 38$ MAPK was dual-phosphorylated during ischemia (Fig. 3) and was inhibited by pre-treatment with $5-\mathrm{mg} / \mathrm{ml}$ Aquilaria extract. The results suggested that p38 MAPK is activated during myocardial ischemia and could be inhibited by 5 $\mathrm{mg} / \mathrm{ml}$ Aquilaria extract.

\section{DISCUSSION}

Aquilaria crassna has been used in many traditional therapeutic purposes and known to be a major composition in traditional Thai herbal formulation (Suvitayavat et al., 2005). Recently, we demonstrated the underline mechanism of antiinflammatory effect of the ethyl acetate extract of A. crassna by attenuating p38 MAPK activation (Kumphune et al., 2011). Avariety of studies have demonstrated that the dual phosphorylation of p38 MAPK occurring during myocardial ischemia is reduced in the presence of p38 MAPK inhibitor (Kumphune et al., 2010), which pointed the cardioprotective effect of p38 MAPK inhibition. Therefore, we hypothesized that the ethyl acetate extract of A. crassna could possibly have cardioprotective effect.

The major finding in this manuscript is that the ethyl acetate extract of $A$. crassna protect the heart from myocardial ischemia/reperfusion injury in isolated murine heart model, and this cardioprotective effect of the extract is ,at least in part, inhibited p38 MAPK phosphorylation, which is the major 
signaling known to aggravate myocardial cell injury and death. Pre-treatment with $5-\mathrm{mg} / \mathrm{ml}$ ethyl acetate extract of $A$. crassna for $30 \mathrm{~min}$ prior to global ischemia/reperfusion significantly reduced the infarct volume. In addition, the same concentration of the extract could also inhibit p38 MAPK dual-phosphorylation.

In our hands, this is the first evidence showing the ex vivo anti-ischemic effect of this A. crassna extract, on isolated murine hearts. The results were consistent with our findings in an in vitro model of cardiac cell, H9c2 (Jermsri et al., $2012^{\mathrm{a}}$ ), as well as isolated adult rat ventricular myocytes (ARVMs) (Kumphune et al., 2012 ${ }^{\mathrm{b}}$ ), that $5 \mathrm{mg} / \mathrm{ml}$ of the extract could reduce simulated ischemia-induced cellular injury and death. Moreover, the same concentration of the extract also inhibited ischemia induced-p38 MAPK activation. However, the experiments in model still have some limitations and weak points, as it may not closely related to real physiological settings in the intact heart. Therefore, the more relevant models, such as an in vivo experiment in animal model, will provide some more beneficial and reliable functional data. The intensive study in large animal model such as rabbit, pig, dog, or etc., should be performed and could provide more functional data related to human heart, in term of heart size, heart volume, hemodynamic parameters. Moreover, this report was performed using the crude extract, so identification of active compounds is still needs to be further investigated.

\section{CONCLUSIONS}

The ethyl acetate extract of $A$. crassna contain antiischemic activity against myocardial ischemia/reperfusion injury. The cardioprotective mechanism of the extract could, at least in part, explained by attenuation of p38 MAPK phosphorylation, which is the major signaling pathway involve in cardiac cell injury and death.

\section{ACKNOWLEDGEMENT}

This work was supported by Naresuan University Research endowment Fund (R2555C068).

\section{REFERENCES}

Braunwald E. Acute myocardial infarction-the value of being prepared. N Eng1 J Med,1996; 334:51-52

Dash M, Patra JK \& Panda PP. Phytochemical and antimicrobial screening of extracts of Aquilaria agallocha Roxb. African Journal of Biotechnology, 2008; 7: 3531-34.

Jennings RB \& Reimer KA. The cell biology of acute myocardial ischemia. Annu Rev Med, 1991; 42: 225-46.

${ }^{\mathrm{a}}$ Jermsri P, Jiraviriyakul A, Unajak S \& Kumphune S. Effect of Aquilaria crassna crude extract on simulated ischemia induced cardiac cell death. Inter J Pharma Bio Sci, 2012; 3: 604 - 13.

${ }^{\mathrm{b}}$ Jermsri P \& Kumphune S. Ethyl Acetate Extract of Aquilaria Crassna Preserve Actin Cytoskeleton on Simulated Ischemia Induced Cardiac Cell Death. J Med Plant Res, 2012; 6: 4057-62.

Kumphune S, Bassi R, Jacquet S, Sicard P, Clark JE, Verma S, Avkiran M, O'Keefe SJ \& Marber MS. A chemical genetic approach reveals that p38 MAPK alpha MAPK activation by diphosphorylation aggravates myocardial infarction and is prevented by the direct binding of SB203580. J Biol Chem, 2010; 285: 2968-75.

Kumphune S, Prompunt E, Phaebuaw K, Sriudwong P, Pankla R \& Thongyoo P. Anti-inflammatory effects of ethylacetate extract of Aquilaria crassna inhibit LPS-induced Tumor Necrosis Factor Alpha production by attenuating p38 MAPK activation. Inter J Green Pharm, 2011; 5: 43-48.

${ }^{a}$ Kumphune S, Chattipakorn S \& Chattipakorn N. Role of p38 MAPK inhibition in cardiac ischemia/reperfusion injury. Eur J Clin Pharmacol, 2012; 68: 513-24.

${ }^{\mathrm{b}}$ Kumphune S, Jermsri P \& Paiyabhroma N. An in vitro antiischemic effect of ethyl acetate extract of Aquilaria crassna in isolated adult rat ventricular myocytes subjected to simulated ischemia. Journal of Phytotherapy and Pharmacology, 2012; 5: 47-54.

Miniyar PB, Chitre TS, Karve SS, Deuskar HJ \& Jain KS. Antioxidant activity of ethyl acetate extract of Aquilaria agallocha on nitriteinduced methemoglobin formation. Inter J Green Pharm, 2008; 2: 43-44.

Suvitayavat W, Tunglert S, Thirawarapan SS \& Bunyapraphatsara N. Effects of Ya-hom on blood pressure in rats. J Ethnopharmacol, 2005; 97: 503-8.

\section{How to cite this article:}

Chanyatip Suwannasing, Nitchawat Paiyabhroma, Sarawut Kumphune. Anti-ischemic effect of ethyl acetate extract of Aquilaria crassna by attenuation of p38-MAPK activation. J App Pharm Sci. 2012; 2 (10): 026-030. 